\title{
Static Data Race Detection for Concurrent Programs with Asynchronous Calls
}

\author{
Vineet Kahlon \\ NEC Labs America, Princeton, USA.
}

\author{
Nishant Sinha \\ NEC Labs America Princeton, USA. \\ Erik Kruus \\ NEC Labs America Princeton, USA.
}

\author{
Yun Zhang \\ Department of Computer Science \\ Princeton University Princeton, USA.
}

In this paper, we present a method to perform static data race detection for concurrent $\mathrm{C}$ programs which use asynchronous indirect function calls for communication. Given a multi-threaded program with asynchronous calls, our method first builds a precise contextsensitive concurrent control flow graph (CCFG) based on a flow and context-sensitive (FSCS) points-to analysis. Using this CCFG, we perform a staged data race detection, that involves (i) identifying the shared variables and lock pointers, (ii) computing an initial database of race warnings, and finally, (iii) pruning away the spurious warnings using a may-happen-in-parallel (MHP) analysis based on computing lock sets and performing thread order analysis. The main drawback of static analysis is that a large number of bogus data race warnings can often be generated which do not correspond to true bugs. Thus a key challenge in static data race detection lies in satisfying the conflicting goals of scalability and accuracy, i.e., keeping the bogus warning rate low.

Our computation model comprises a thread pool, where each thread iteratively processes tasks from its work queue. In order to send a task request to another thread $t_{2}$, thread $t_{1}$ issues an asynchronous function call with the task (e.g., a function to be executed) as an argument. An asynchronous function call may or may not be followed by an asynchronous function return (join). Note that the standard fork-join model for concurrent programs can be viewed as a particular case of the thread pool model (with unbounded number of threads, each having a zero-length queue), where fork corresponds to an asynchronous function call (AFC) to an unnamed thread while the join corresponds to the matching asynchronous return. When the thread pool is of finite size, each thread must have a non-zero length queue to store multiple incoming job requests. A key challenge in analyzing such programs is that given an asynchronous call, the function to be executed during the call, the arguments to that function, the callback functions passed to the call and the thread on which the call is to be executed can all be passed indirectly via pointers. Moreover, the values of each of these pointers can be set upstream in the code far away from the function call location. Thus without a precise (flow and context-sensitive) pointsto analysis it is hard to even determine control flow in the given program. Recursion further complicates the problem.

The first and most crucial step in static data race detection, therefore, is to construct the CCFG of the given program. In the presence of function pointers and recursion, however, constructing the CCFG of the given program that satisfies the three key criteria of (i) Soundness: preserving every real data race, (ii) Accuracy: keeping the bogus warning rate low, and (iii) Scalability, is a nontrivial problem.

The main problem posed by the presence of function pointers is that it is not possible to compute the CCFG from the syntactic program description. In order to resolve these function pointers while keeping the bogus warning rate low, we have to carry out a flow and context sensitive (FSCS) function pointer alias analysis. 
For FSCS pointer alias analysis we, in turn, need to compute the CCFG thereby creating a cyclic dependency between CCFG construction and function pointer resolution. This cyclic dependency can be broken via a context-sensitive construction of the CCFG wherein the contexts of the given program are enumerated starting at the entry function. As soon as a function pointer is encountered in a given context, its points-to set is computed which allows us to continue constructing the CCFG. A context-sensitive CCFG construction presents no problems in the absence of recursion. If recursion is present, however, the number of calling contexts for a recursive function can, in principle, be infinite. The may result in the size of the context sensitive CCFG being infinite. In [6], the problem is handled by introducing back edges for recursive functions. Thus if during the construction of the CCFG, the same function is encountered again in a given context, then instead of introducing a new copy of $f$ a back edge is introduced to the existing copy of $f$. However, because of the introduction of back edges the construction no longer remains truly context-sensitive thereby leading to an over-approximation of the set of behaviors of the given program. This, in turns, leads to an increase in bogus data race warnings (see Sec. 6) rendering this technique unsuitable for our purposes.

In this paper, we present a new, unified approach for constructing the CCFG of a concurrent program with asynchronous calls, recursion and function pointers, while ensuring the precision of data race detection. The apparently disjoint goals of context-sensitive CCFG construction (using function pointer analysis) and lockset computation are achieved simultaneously by a uniform data flow analysis with a fix-point criteria. First, our analysis dove-tails the context-sensitive construction of the CFG with the (contextsensitive) computation of the points-to sets of function pointers. This allows us to resolve the functions called via pointers as and when they are encountered during the construction process. Secondly, in order to ensure that the size of the resulting CFG is finite, we formulate a fix-point criterion which enables us to enumerate a representative finite set of contexts that need be explored while ensuring both soundness and accuracy of static data race detection. The key insight in this work is that for purpose of static data race detection, it suffices to consider only those contexts that may generate different locksets at locations where shared variables are accessed. Such contexts are only finitely many in number. Interestingly, our fix-point criteria not only ensures finiteness of CCFG, but also prunes out contexts which cannot generate new data race warnings (even for program without function pointers and/or recursion - see Section 8). A fundamental difference between our technique and [6] is that in (over-)approximating all possible contexts via back edges the analysis in [6] loses precision and no longer remains truly context-sensitive. This directly impacts the accuracy of computing the context sensitive locksets leading to bogus warnings. We, on the other hand, use a fix-point criterion to explicitly enumerate a finite subset of contexts that need be explored while preserving soundness, i.e., no data race is missed, and carry out a precise context-sensitive lockset analysis individually for each of these contexts.

An additional challenge that needs to be addressed when computing FSCS points-to sets of lock and function/thread pointers is scalability. Indeed, whole program FSCS alias analysis is expensive. In order to ensure scalability, we leverage bootstrapping [11]. Since, for our application, we are interested only in lock and function/thread pointers we need to consider only those program statements which may affect aliases of these pointers. These statements can be isolated via bootstrapping. Since the statements that may affect aliases of lock or function pointers are very few in number it guarantees scalability of the FSCS analysis for lock and function/thread pointers. To further enhance scalability, we leverage the use of summarization. Since lock and function pointer aliases are typically updated by only a few functions, summaries for FSCS alias analysis (as formulated in [11]) for these pointers need be computed for only a small number of functions. Thus exploiting the synergy between bootstrapping and summarization enables us to efficiently compute the FSCS aliases of lock and function pointers during the CCFG construction.

Our approach has been implemented in the CoBE framework [12] for analyzing multi-threaded C programs. Section 2 presents a motivating example program which combines thread and indirect asynchronous call models. Section 3 describes the background concepts and notations used in the paper. We present the algorithm for constructing concurrent control flow graphs in Section 6. Based on the CCFG created, we present our technique for computing and refining the set of data race warnings in Section 7. Finally we describe the related work and conclude in Section 9.

\section{Motivating Example}

Figure 1 shows a slice of a concurrent $\mathrm{C}$ program which illustrates some of the complexities arising from a combination of indirect function calls and thread creation.

We use C-like syntax together with a special thread construct to denote thread identifiers and named fork (see Section 3 ) and join calls to denote thread creation and termination. More precisely, the call fork $(t 1, f, \arg 1, \arg 2, \ldots)$ denotes the creation of a thread with identifier $t 1$ which executes the function $f$ with the actual arguments arg1, arg2, etc. . Similarly the call join (t1) blocks the caller until the thread $t 1$ finishes execution.

The example program starts from the main function, which creates threads t1 or t2 (lines 10, 11 respectively). Both the threads execute the function $f$; however, depending on the arguments passed to $f$, the callback function $h 1$ or $h 2$ is invoked. The function $f$ obtains the return value from the callback function and then writes to a shared variable $* z$. Since the shared variable may be written in either thread $* \mathrm{t} 1$ or $* t 2$, there is a potential race condition at the locations 12 and 13 in function $f$. However, as we show later, if we build the concurrent call graph in a contextsensitive manner and take into account the thread ordering imposed by thread creation and join, we can prove that such a race cannot happen. Also, note that since the functions $\mathrm{h} 1$ and $\mathrm{h} 2$ are called using function pointers, we will not be able to capture the exact asynchronous behavior of the program without a precise function pointer analysis.

\section{Preliminaries}

In this paper, we focus on static data race detection of multithreaded C programs. A popular paradigm for writing multithreaded C programs is the POSIX-style fork/join model. Here the program is comprised of a main thread which may fork one or more new threads providing them with a function pointer argument pointing to the function that the new thread must execute. In case, a thread executing a fork call needs to wait for the call to finish a matching join is executed.

While such a model is adequate for small programs, for efficiency reasons or due to resource limitations, large industrialstrength applications often go beyond this simple thread creation model by adopting a more flexible thread pool model [28], consisting of a number of threads each having its own work queue for processing tasks. In many cases, a set of threads are created at initialization and each thread is named, i.e., it has an unique identifier. Since the threads are created once at initialization, the caller thread simply creates an execution job and dispatches it to the callee thread. In order to send a task request to another thread $t_{2}$, thread $t_{1}$ issues an asynchronous function call $(A F C)$ with the task (e.g., a function to be executed) as an argument. Note that the standard 


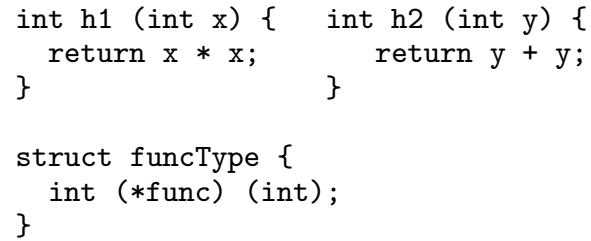

Figure 1. Motivating example concurrent program.

fork-join model for concurrent programs can be viewed as a particular case of the thread pool model (with unbounded number of threads, each having a zero-length queue), where fork corresponds to an AFC to an unnamed thread while the join corresponds to the matching asynchronous return. When the thread pool is of finite size, each thread must have a non-zero length queue to store multiple incoming job requests.

Program Model: Concurrent Control Flow Graph (CCFG) We consider concurrent imperative programs comprising threads that communicate using shared variables and synchronize with each other using standard primitives such as locks. Each thread $T_{i}$ : $\left(F_{i}, \mathrm{e}_{i}, G_{i}, L_{i}\right)$ consists of procedures $F_{i}$, entry procedure $\mathrm{e}_{i} \in F_{i}$, a set of global variables $G$ and thread local variables $v$. Each procedure $p \in F$, is associated with a tuple of formal arguments $\operatorname{args}(p)$, a return type $t_{p}$, local variables $L(p)$, and a control flow graph (CFG). Each procedural CFG $(N(p), E(p)$, action) consists of a set of nodes $N(p)$ and a set of edges $E(p)$ between nodes in $N(p)$. A node in $N(p)$ is designated the entry node of the procedure and represents the statement wherein control flow enters the procedure. Similarly a subset of nodes of $N(p)$ are designated the exit nodes and represent statements where control flow may leave the procedure. Each edge $m \rightarrow n \in E(p)$ is associated with an action that is an assignment, a call to another procedure, a return statement, a conditional guard, a synchronization statement, a named fork statement or a named join statement. The actions in the CFG for a procedure $p$ may refer to variables in the set $G \cup$ $\operatorname{args}(p) \cup L(p)$. The named fork edge provides a means to model, in a unified fashion, the fork operation in the fork/join model and AFCs in the thread pool model. A named fork edge occurs from the program location where the fork or AFC is made to the entry node of the procedure to be executed. If the thread on which the procedure is to be executed is specified as in the thread pool model, the named fork is labeled with the corresponding thread-id. If the thread-id is unknown or irrelevant as in the fork/join model it is simply labeled with '?'. A named join matching a named fork that executes procedure $g$ occurs from an exit node of $g$ to the node representing the join location.

Complexities of AFCs. While the AFC construct provides a very flexible and powerful tool in the hands of programmers, it also introduces many challenges when it comes to analyzing code. A common mechanism to implements AFCs is via the use of bound function objects, e.g., in the Boost library [23]. For example, the function makeAFC creates an AFCTask bf in the following way.

struct AFCTask $*$ bf $=$ makeAFC (thread_t, bind (\&g,args));

The bf object contains the thread pointer thread_t, i.e., the thread on which the task is to be executed, the function pointer $\& g$ pointing to the function $g$ to be executed, and args are the arguments to $g$. The arguments args are bound to the corresponding function $g$ using the bind construct. The actual asynchronous function call takes place with the help of the enqueue function: on executing enqueue(bf) in the caller thread, the bf object task is dispatched to the working queue of the thread pointed-to by thread_t, which is then executed in a first-in-first-out manner from the queue. Note that the location of creation and dispatch of an AFC may be far removed from each other in the code. Liberal use of AFCs can lead to code that is extremely hard to understand and debug. Consider, for example, the following nested AFC object af c, where the function object created by bind (\&bar, barArg) is itself an argument to another function object:

struct AFCTask *afc = makeAFCTask ( $t$, bind (\&g, gArg1, gArg2, bind (\&bar, barArg)) );

On dispatching the above object, the task with function $g$ (and arguments $g A r g 1, g A r g 2$ and $\operatorname{bind}(\& b a r, b a r A r g)$ ) will be executed on thread $t$. Upon completion, $g$ may make another AFC with function bar and argument barArg. The target of the second AFC might be the same thread $t$ or another thread whose identifier is available to $g$ at the time of execution. Note that this target was kept ambiguous at the time of creation of the afc object, and the intent of the programmer at the creation point might be completely forgotten while implementing the function $g$. This gives rise to subtle bugs which produce unexpected execution results and are extremely difficult to trace due to the indirect call sequences. Since the instantiations to the various entities in an AFC, i.e., thread pointers, function pointers, arguments, etc., may be physically spread out in the source code, it is easy to see that one of the key challenges in analyzing multi-threaded $\mathrm{C} / \mathrm{C}++$ code with AFCs lies in constructing the CCFG from source code.

\section{Bootstrapping based Pointer Analysis}

We review some useful facts about the well-known Steensgaard's points-to analysis [24] and how to leverage it in improving the scalability of flow and context-sensitive (FSCS) alias analyses via bootstrapping [11].

Steensgaard's Analysis. In Steensgaard's analysis [24], aliasing information is maintained as a relation over abstract memory locations. Every location $l$ is associated with a set of pointers and holds some content $\alpha$ which is an abstract pointer value. Points-to information between abstract pointers is stored as a points-to graph which is a directed graph whose nodes represent sets of pointers and edges encode the points-to relation between them. Intuitively, an edge $e: v_{1} \rightarrow v_{2}$ from nodes $v_{1}$ to $v_{2}$ represents the fact that a pointer in the set represented by $v_{1}$ may point to some pointer 
in the set represented by $v_{2}$. The key feature of Steensgaard's analysis that is used in bootstrapping is the well known fact that the points-to sets so generated are equivalence classes (see [11]). Hence these sets define a partitioning of the set of all pointers in the given program into disjoint subsets, called Steensgaard Partitions, that respect the aliasing relation, i.e., a pointer can only be aliased to pointers within its own partition. For pointer $p$, let $n_{p}$ denote the node in the Steensgaard points-to graph representing the Steensgaard partition containing $p$. A Steensgaard points-to graph defines an ordering on the pointers in $P$ which we refer to as the Steensgaard points-to hierarchy (see [11]). For pointers $p, q$, we say that $p$ is higher than $q$ in the Steensgaard points-to hierarchy, denoted by $p>q$, or equivalently by $q<p$, if $n_{p}$ and $n_{q}$ are distinct nodes and there is a path from $n_{p}$ to $n_{q}$ in the Steensgaard points-to graph. Also, we write $p \sim q$ to mean that $p$ and $q$ both belong to the same Steensgaard partition.

Divide and Conquer via Bootstrapping. Whole program flow sensitive and context sensitive (FSCS) alias analysis is expensive. However, for many applications we require FSCS aliases for only a small set of pointers of interest. For instance, for static data race detection, we need to compute FSCS aliases for only the lock pointers. Thus, if we are interested in computing the aliases of pointers in a given set $S$, we want to leverage divide and conquer by restricting our analysis only to those statements of the given program that may affect aliases of pointers in $S$. Towards that end, we leverage bootstrapping [11] which exploits the fact that the aliases of a pointer in a Steensgaard partition $P$ can be affected only by assignments to either a pointer in $P$ or a pointer $q$ higher in the Steensgaard points-to hierarchy than some pointer in $P$. Assume now that our goal is to compute FSCS aliases of a pointer $p \in P$. Then it suffices to restrict our analysis only to statements that directly modify values of pointers in the set $P_{\geq}$comprised of all pointers $q$ such that either $q>p$ or $q \sim p$. It follows from the above observation that if we are interested in the FSCS aliases of pointers in a set $S$, then it suffices to restrict the analysis to pointers in the Steensgaard-closure of $S$ defined as follows:

Definition (Steensgaard Closure). The Steensgaard closure of a set $S$ of pointers, denoted by $C l(S)$, in the minimal set with the property that $S \subseteq C l(S)$ and for each $p \in C l(S)$ if either $q \sim p$ or $q>p$ then $q \in C l(S)$.

\subsection{Complete Update Sequences}

In resolving FSCS points-to sets of function/thread pointers during CCFG construction, we exploit summarization in which the notion of a complete update sequence plays a crucial role. A pointer $p$ is said to be semantically equivalent to $q$ at location $l$ if $p$ and $q$ have the same value at $l$ (even if they are syntactically different).

Definition (Complete Update Sequence) [11]. Let $\lambda: l_{0}, \ldots, l_{m}$ be a sequence of successive program locations and let $\pi$ be the sequence $l_{i_{1}}: p_{1}=a_{0}, l_{i_{2}}: p_{2}=a_{1}, \ldots, l_{i_{k}}: p_{k}=a_{k-1}$, of pointer assignments occurring along $\lambda$. Then $\pi$ is called a complete update sequence from $p$ to $q$ leading from locations $l_{0}$ to $l_{m}$ iff

- $a_{0}$ and $p_{k}$ are semantically equivalent to $p$ and $q$ at locations $l_{0}$ and $l_{m}$, respectively.

- for each $j, a_{j}$ is semantically equivalent to $p_{j}$ at $l_{i_{j}}$,

- for each $j$, there does not exist any (semantic) assignment to pointer $a_{j}$ between locations $l_{i_{j}}$ and $l_{i_{j+1}}$; to $a_{0}$ between $l_{0}$ and $l_{i_{1}}$; and to $p_{k}$ between $l_{i_{k}}$ and $l_{m}$ along $\lambda$.

A related concept is that of maximally complete update sequences.

Definition 4 (Maximally Complete Update Sequence). Given a sequence $\lambda: l_{0}, \ldots, l_{m}$ of successive control locations starting at the entry control location $l_{0}$ of the given program, the maximally complete update sequence for pointer q leading from locations $l_{0}$ to $l_{m}$ along $\lambda$ is the complete update sequence $\pi$ of maximum length, over all pointers $p$, from $p$ to $q$ (leading from locations $l_{0}$ to $l_{m}$ ) occurring along $\lambda$. If $\pi$ is an update sequence from $p$ to $q$ leading from locations $l_{0}$ to $l_{m}$, we also call it a maximally complete update sequence from $p$ to q leading from locations $l_{0}$ to $l_{m}$.

Maximally complete update sequences can be used to characterize aliasing.

Theorem [11] (Aliasing Theorem) Pointers $p$ and $q$ are aliased at control location l iff there exists a sequence $\lambda$ of successive control locations starting at the entry location $l_{0}$ of the given program and ending at $l$ such that there exists a pointer a with the property that there exist maximally complete update sequences from a to both $p$ and $q$ (leading from $l_{0}$ to $l$ ) along $\lambda$.

Advantages of using Update Sequences. A key advantage of using update sequences to characterize aliasing is that update sequences can be summarized in a compact manner. Additionally, bootstrapping allows us to exploit locality of reference. Indeed, since Steensgaard partitions are typically small, by restricting summary computation to each individual partition ensures that the resulting summaries will also be small. Secondly, the number of statements modifying values of pointers in a given partition also tend to be few and highly localized to a few functions. This in turn, obviates the need for computing summaries for functions that don't modify any pointers in the given partition which typically accounts for majority of the functions. Note that without partitioning it would be hard to ensure viability of the summarization approach. Thus it is the synergy between divide and conquer and summarization that ensures scalability of the FSCS alias analysis.

\section{Static Data Race Detection}

The classical approach to data race detection involves five steps.

1. Identify shared variables, i.e., variables which can be accessed by two or more threads.

2. Enumerate control locations where shared variables are read or written. These determine potential locations where data races can arise.

3. Determine locksets, i.e., the set of locks held, at locations where shared variables are accessed.

4. Each pair of control locations in two different threads where the same shared variable is accessed and disjoint sets of locks are held is labeled a data race warning.

5. Use causality constraints imposed by fork/join operations as well as synchronization primitives to reduce the set of warnings.

Context-Sensitive Lockset Analysis Since locks are typically accessed via pointers, in order to determine locksets in step 3, a precise points-to analysis must be carried out. Consider, a data race warning $\left\langle\left(c_{1}, L_{1}\right),\left(c_{2}, L_{2}\right)\right\rangle$, where $c_{1}$ and $c_{2}$ indicate control locations in two different threads where the same shared variable is accessed with at least one of the accesses being a write operation and $L_{1}$ and $L_{2}$ are the locksets at $c_{1}$ and $c_{2}$, respectively, such that $L_{1} \cap L_{2}=\emptyset$. Note that since in static analysis we typically ignore conditional statements, all syntactic paths leading to location $c_{i}$ are possible. Thus $L_{i}$ must capture the set of locks that must be held irrespective of the path leading to $c_{i}$, i.e., $L_{i}$ must be the intersection of locks held along all paths leading to $c_{i}$, often referred to in the literature as must-locksets. For the sake of accuracy it is, therefore, imperative that points-to sets of lock pointers be computed context sensitively. This is because lock pointers typically point-to different locks in different contexts so that a context-insensitive lockset computation would result in the must-locksets being empty at most 


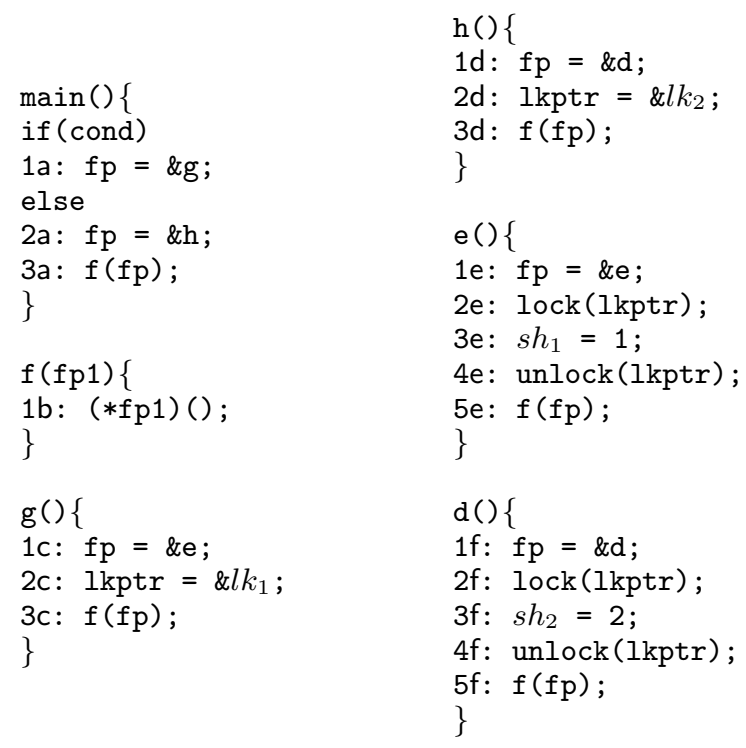

Figure 2. An Example Program

locations where shared variables are accessed. This would lead to an explosion in the number of bogus warnings.

\section{Concurrent Control Flow Graph Computation}

In order to carry out static data race detection via steps 1-5 enumerated above we need to first construct the concurrent control flow graph of the given program. In the absence of function pointers and asynchronous calls this is straightforward. However, the presence of recursion and function/thread pointers give rise to several challenges discussed below.

Function and Thread Pointers. Realistic programs often make use of (indirect) function calls via function pointers and asynchronous calls involving function and thread pointers (see Sec. 3). As a result, it is not possible to compute the call graph of a program from its syntactic program description. In order to resolve these function/thread pointers we have to carry out a function/thread pointer alias analysis. Additionally, for must-lockset computation (see sec. 5), we need to compute the points-to sets of lock pointers context-sensitively for which the contexts of the given program have to be enumerated precisely. In order to enumerate contexts, i.e., construct the CCFG, the points-to sets of the function/thread pointers need to be computed context sensitively which, in turn, requires us to first compute the CCFG. This creates a cyclic dependency between CCFG construction and resolution of function/thread pointers.

Callback Functions. Callback functions are often passed as arguments to function calls, e.g., for exception handling. These callback functions, which are usually passed via function pointers, may be initialized much before the function call executes. Therefore, we must track the values of function pointer arguments for each function call in order to build the CCFG. An additional challenge in that the values of function pointers, in many cases, depend on the actual calling context of the called function. Thus the function pointer points-to analysis for resolving callback function pointers needs to be both flow and context-sensitive.

Recursion. As noted above, in order to resolve points-to sets of function pointers, it is important to carry out a context-sensitive points-to analysis. Because of the cyclic dependency between CCFG construction and resolution of points-to sets of function pointers, we need to construct the CCFG context-sensitively. To- wards that end, an obvious approach is to enumerate all the contexts of the given program starting from the entry function. As soon as a function pointer is encountered in a given context, its points-to set is computed which allows us to continue constructing the CCFG. Note that since we resolve the points-to sets of function pointers in a given context, we can usually resolve the function being called uniquely. A context-sensitive CCFG construction presents no problems in the absence of recursion. In the presence of recursion, however, the number of calling contexts for a recursive function $f$ can, in principle, be infinite. This may result in the size of the context sensitive CCFG being infinite. In [6], the problem is handled by introducing back edges for recursive functions. Thus if during the construction of the CCFG, the same function is encountered again in a given context, then instead of introducing a new copy of $f$ a back edge in introduced to an existing copy of $f$. However, the introduction of back edges over-approximates the behaviors of the given program as a result of which the construction no longer remains context-sensitive (see below for an example). This reduces the accuracy of the must-lockset computation which, as discussed above, needs to be context-sensitive, thereby leading to bogus data race warnings.

Scalability. Finally, since we need to carry out a FSCS points-to analysis to compute the points-to sets of the function, thread and lock pointers, we need to contend with scalability issues.

\subsection{CCFG Construction}

We start by presenting an algorithm for constructing the concurrent control flow graph (CCFG) that handles the issues discussed above while ensuring both scalability as well as accuracy of the overall static data race detection framework. As discussed above, the cyclic dependency between CCFG construction and resolution of the points-to sets of function pointers can be broken by constructing the CCFG context-sensitively and resolving the points-to sets of the function pointers on-the-fly. The main challenge in following this approach lies in handling recursion. If there exist recursive procedures in the given program then it may, in principle, result in infinitely many contexts. Indeed, a self-recursive function $f$ with a function call $f$ call to itself will generate the infinitely many contexts fcall $^{i}$, where $i \geq 1$.

In order to resolve this problem, in [6] if while constructing the CCFG a function $f$ is encountered again in a given context, then a back edge to an existing copy of $f$ is introduced. This approach, however, is not suitable for static data race detection as it leads to bogus warnings as we now illustrate.

Example. Consider a concurrent program comprised of two threads each running the code shown in Fig. 2 with main as the entry function and $s h_{1}$ and $s h_{2}$ being the shared variables. The algorithm for CFG construction formulated in [6] builds an invocation graph by unrolling the contexts, i.e., sequence of function calls of the given program. For our example, the procedure starts with the entry function main. Since main calls $f$, an edge is added from main to $f$ in the invocation graph. In $f$, the function pointer $f p l$ could point to either $g$ or $h$, depending upon whether cond evaluates to true or false in main. Thus both $g$ and $h$ are added as successors to $f$. Function $g$, in turn, calls $f$. Since $f$ already exists in the current context, i.e., main $\rightarrow f \rightarrow g$, a back edge to $f$ is added in the invocation graph. Similarly a back-edge is added from $h$ to $f$. Finally, $f$ may call $e$ and $d$ both of which, in turn, call $f$. Thus $e$ and $d$ are both added as successors to $f$ along with back edges from $e$ and $d$ to $f$ resulting in the invocation graph shown in fig 3(a).

Because of the structure of the resulting invocation graph, we see that the must-locksets of $l k p t r$ at locations $2 \mathrm{e}$ and $2 \mathrm{f}$ in functions $e$ and $d$, respectively, are empty sets. This is because $l k p t r$ is set as pointing-to $l k_{1}$ and $l k_{2}$ in functions $g$ and $h$, respectively. 


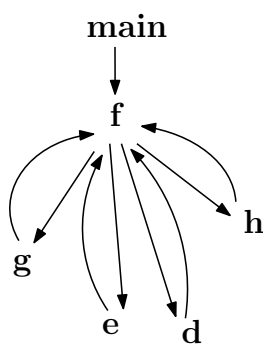

(a)

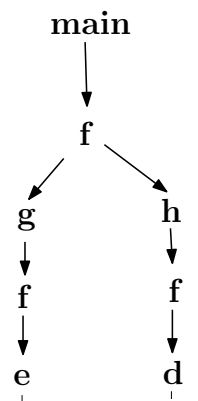

(b)
Figure 3. Call graph for the example in Fig. 2

In computing the must-aliases of $l k p t r$ at 2 e, we must, by definition, take the intersection of aliases of $l k p t r$ along all paths in the invocation graph leading to $2 \mathrm{e}$. However, due to the existence of back-edges from both $g$ and $h$ to $f$, we see that the paths main, $f$, $g, f, e$ and main, $f, h, f, e$ contribute different aliases, i.e., $\& l k_{1}$ and $\& l k_{2}$, respectively. Thus the set of must-aliases of $l k p t r$ at $2 e$ is the empty set. As a result, the must-locksets at $3 e$ and $3 f$ are empty sets. In a concurrent program with two threads executing the code shown in Fig. 2, this would result in the pair $(3 \mathrm{e}, 3 \mathrm{e})$ being labeled a data race warning. Similarly, (3f, 3f) would also constitute a data race warning.

If, however, we unroll the contexts without introducing back edges we get an infinite call graph, part of which is shown in fig. 3(b). Here we see that starting at the entry function main, all paths leading to any given instance of $e$ pass only through $g$ without passing through $h$. Thus we can uniquely resolve the points-to set of $l k p t r$ at $2 \mathrm{e}$ as $l k_{1}$ as a result of which the must-lockset at $3 \mathrm{e}$ is $\left\{l k_{1}\right\}$. Similarly, the lockset at $3 f$ is $\left\{l k_{2}\right\}$. Since the same lock is held at $3 \mathrm{e}$ in both the threads, $(3 \mathrm{e}, 3 \mathrm{e})$ no longer constitutes a data race warning. Similarly, $(3 f, 3 f)$ is also not a data race warning.

To sum up, introducing back-edges in the invocation graph overapproximates the set of behaviors of the given program leading to bogus warnings. Thus finitizing the CCFG via the introduction of back edges is not a viable technique for static data race detection. We now present a new technique that instead of (over)approximating all possible contexts via back-edges, enumerates a finite subset of contexts that need be explored without losing precision of the analysis at hand. Once these contexts have been enumerated, the must-locksets can be computed in each individual context thereby preserving context-sensitivity in lockset computation that is lost in the back-edge approach.

\subsection{Lockset-based Fix-Point Procedure}

In the presence of recursion, the number of contexts in a program is infinite. However, for our application, e.g., lockset-based static data race detection, by exploiting the fact that not all these contexts may generate different data race warnings, we show that it suffices to explore only finitely many contexts. Broadly speaking, our goal is to consider only those contexts that may generate different locksets at locations where shared variables are accessed.

CCFG Finitization: Our core strategy for finitizing the CCFG is to explore only those contexts in which the points-to sets of lock, function or thread pointers, or locksets are different. Note that lock pointers need to be tracked for lockset computation for data race detection whereas function and thread pointers need to be tracked for constructing the CCFG.

In general, tracking points-to sets of all pointers in the given program in a flow and context-sensitive (FSCS) fashion would be intractable. For our application, however, since we need to track points-to sets of only the set $S$ of lock, function and thread pointers, we can leverage bootstrapping [11]. Towards that end, we note that the Steensgaard closure $C l(S)$ of $S$ (see Sec. 4) would simply be the set of lock, function and thread pointers, pointers to lock, function or thread pointers, pointers to pointers to lock, function or thread pointers, etc. Thus when computing FSCS aliases of the set $S$, we can slice away all statements of the given program that do not affect the aliases of pointers in $S$, i.e., those that are not assignments to any pointer in $C l(S)$. Since the number of statements affecting lock, function or thread pointers is typically small, bootstrapping results in a highly precise as well as scalable FSCS analysis for such pointers.

In computing the CCFG context sensitively, if we encounter a function call again, we need to decide whether to explore it or not. Let $\operatorname{con}_{1}=\operatorname{con}_{1}^{\prime} . f c a l l_{1}$ and $\operatorname{con}_{2}=\operatorname{con}_{2}^{\prime} . f c a l l_{2}$ be two contexts such that $f_{\text {call }}$ and $f$ call $_{2}$ are instances of the same function call. Suppose that in constructing the CCFG we have already explored the context $\mathrm{con}_{1}$ and then encounter the context $\operatorname{con}_{2}$. Our criterion for continuing to explore $\mathrm{fcall}_{2}$ is whether doing so could lead to the discovery of new aliases for lock, function or thread pointers or new locksets. Towards that end, we show that if in the two contexts $\operatorname{con}_{1}^{\prime}$ and $\operatorname{con}_{2}^{\prime}$ the aliases of all pointers in $C l(S)$ are the same then exploring $\mathrm{fcall}_{2}$ further cannot lead to the discovery of new alias sets at any given location for any pointer in $C l(S)$. This follows from the following result (see [26] for the proof).

Theorem 1 (Finitization). Let $R$ be a set of pointers and let $C \mathrm{Con}_{1}=\operatorname{con}_{1} \cdot f c_{1}$ and $C_{\mathrm{Con}}=\mathrm{con}_{2} \cdot f c_{2}$ be contexts such that $f c_{1}$ and $f c_{2}$ are instances of the same function call $f$. Suppose that (i) for each $p \in C l(R)$, the aliases of $p$, and (ii) the must-locksets, are the same at the call location of $f c$ in both the contexts con $_{1}$

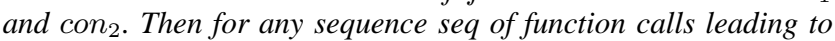
function $h$, if Con ${ }_{1}^{\prime}=$ Con $_{1}$.seq and Con ${ }_{2}^{\prime}=$ Con $_{2}$. seq are valid contexts then the aliases of each pointer in $C l(R)$ and the mustlocksets are the same at each location of $h$ in $\mathrm{Con}_{1}^{\prime}$ and $\mathrm{Con}_{2}^{\prime}$.

Proof. Let loc be a location of $h$ and let $p \in C l(R)$. Let $q$ be aliased to $p$ at loc in context $C o n_{1}^{\prime}$. We show that $q$ is also aliased to $p$ at $l o c$ in context $\mathrm{Con}_{2}^{\prime}$. Since $p$ and $q$ are aliased to each other in $C o n_{1}^{\prime}$, by the aliasing theorem (see pg. 4), there exist maximal update sequences $\operatorname{seq}_{p}: l_{i_{1}}: p_{1}=a_{0}, l_{i_{2}}: p_{2}=a_{1}, \ldots, l_{i_{k}}: p_{k}=a_{k-1}$ and $\operatorname{seq}_{q}: l_{j_{1}}: q_{1}=b_{0}, l_{j_{2}}: q_{2}=b_{1}, \ldots, l_{j_{m}}: q_{m}=b_{m-1}$ starting from the entry location of the given program and leading to loc along $C o n_{1}^{\prime}$ such that (i) $a_{0}=b_{0}$, (ii) $p_{k}$ and $q_{m}$ are semantically ${ }^{1}$ equal to $p$ and $q$ at locations $l_{i_{k}}$ and $l_{j_{m}}$, respectively, (iii) for each $k^{\prime}, p_{k^{\prime}}$ is semantically equal to $a_{k^{\prime}}$ at $l_{i_{k^{\prime}+1}}$ and $p_{k^{\prime}}$ is not modified (semantically) along the sequence $l_{k^{\prime}}+1, \ldots, l_{k^{\prime}+1}$ of program locations, and (iv) for each $l^{\prime}, q_{l^{\prime}}$ is semantically equal to $b_{l^{\prime}}$ at $l_{l_{l^{\prime}+1}}$ and $q_{l^{\prime}}$ is not modified (semantically) along the sequence $l_{l^{\prime}}+1, \ldots, l_{l^{\prime}+1}$ of program locations. Suppose that along the context $\operatorname{Con}_{1}^{\prime}$, the location $l_{\text {call }}$ from where $f c_{1}$ is called occurs between the locations $l_{i_{c_{1}}}$ and $l_{i_{c_{1}}+1}$, i.e., call $l_{1}$ lies in the open interval $\left(i_{c_{1}}, i_{c_{1}}+1\right)$ (we assume that there is no assignment at the location of a function call). Similarly, we assume that $l_{\text {call }}$ occurs between the locations $l_{j_{c_{2}}}$ and $l_{j_{c_{2}}+1}$ along $\operatorname{Con}_{1}^{\prime}$, i.e., call $_{1} \in\left(j_{c_{2}}, j_{c_{2}}+1\right)$. Note that the location $l_{\text {call }}$ occurs along the context $\operatorname{con}_{1}$.

Consider now the maximal update sub-sequences $s e q_{q}^{\prime}: l_{i_{1}}$ : $p_{1}=a_{0}, l_{i_{2}}: p_{2}=a_{1}, \ldots, l_{i_{1}}: p_{c_{1}}=a_{c_{1}-1}$ and $s e q_{q}^{\prime}: q_{1}=b_{0}$, $l_{j_{2}}: q_{2}=b_{1}, \ldots, l_{j_{c_{2}}}: q_{c_{2}}=b_{c_{2}-1}$. We observe that there cannot occur a statement that (semantically) modifies $p_{c_{1}}$ between locations $l_{i_{c_{1}}}$ and $l_{\text {call }}$ along $\operatorname{con}_{1}$, else $s e q_{p}$ won't be a complete update sequence. Thus $s e q_{p}^{\prime}$ is, in fact, a maximally complete

\footnotetext{
${ }^{1}$ We use the term semantically equal to mean that $p_{k}$ and $q_{m}$ could be accessed directly or indirectly, i.e., via dereferencing of pointer variables.
} 
update sequence from the entry location of the given program to the location $l_{\text {call }}$. Similarly, $s e q_{q}^{\prime}$ is a maximally complete sequence from the entry location of the given program to the location $l_{\text {call }}$ along $\operatorname{con}_{1}$. Then since $a_{0}=b_{0}$, we have by the aliasing theorem, that $p_{c_{1}}$ and $q_{c_{2}}$ are aliased to each other at location $l_{\text {call }_{1}}$ in $\operatorname{con}_{1}$. Then by the hypothesis of our theorem, $p_{c_{1}}$ and $q_{c_{2}}$ are also aliased to each other at the location $l_{\text {call }_{2}}$ from where $f c_{2}$ is called in $\mathrm{con}_{2}$. From the aliasing theorem, we have that there exist complete maximal update sequences $\mathrm{seq}_{p}^{\prime}: l_{i_{1}^{\prime}}: r_{1}=c_{0}, l_{i_{2}^{\prime}}: r_{2}=c_{1}, \ldots$, $l_{i_{u}^{\prime}}: r_{u}=c_{u-1}$ and $\operatorname{seq}_{q}^{\prime}: l_{j_{1}^{\prime}}: s_{1}=d_{0}, l_{j_{2}^{\prime}}: s_{2}=d_{1}, \ldots$, $l_{j_{v}^{\prime}}: s_{v}=d_{v-1}$ starting from the entry location of the given program and leading to $l_{\text {call }_{2}}$ such that $c_{0}=d_{0}$; for each $k^{\prime}, r_{k^{\prime}}$ is semantically equal to $c_{k^{\prime}}$; for each $l^{\prime}, s_{l^{\prime}}$ is semantically equal to $d_{l^{\prime}}$; and $r_{u}$ and $s_{v}$ are semantically equal to $p_{c_{1}}$ and $q_{c_{2}}$ at $l_{\text {call }}$ respectively. Consider now the sequences, seq $_{p}: l_{i_{1}^{\prime}}: r_{1}=c_{0}$, $l_{i_{2}^{\prime}}: r_{2}=c_{1}, \ldots, l_{i_{u}^{\prime}}: r_{u}=c_{u-1}, l_{i_{c_{1}}+1}: p_{c_{1}+1}=a_{i_{c_{1}}}, \ldots$, $l_{i_{k}}: p_{k}=a_{k-1}$ and $\operatorname{seq}_{q}: l_{j_{1}^{\prime}}: s_{1}=d_{0}, l_{j_{2}^{\prime}}: s_{2}=d_{1}, \ldots$, $l_{j_{v}}: s_{v}=d_{v-1}, l_{j_{c_{2}}+1}: q_{c_{2}+1}=b_{c_{2}}, \ldots, l_{j_{m}}: q_{m}=b_{m-1}$. Note that $r_{u}$ is semantically equal to $p_{c_{1}}$ which, in turn, is semantically equal to $a_{c_{1}}$. Thus $r_{u}$ is semantically equal to $a_{c_{1}}$. Similarly, $s_{v}$ is semantically equal to $b_{c_{2}}$. From this it follows that $\operatorname{seq}_{p}$ and $\operatorname{seq}_{q}$ are maximally complete update sequences starting from the entry location of the given program to location loc in $h$ along $\mathrm{Con}_{2}^{\prime}$. Since $c_{0}=d_{0}$ and $p_{k}$ and $q_{m}$ are semantically equal to $p$ and $q$ respectively, we have, by the aliasing theorem, that $p$ and $q$ are aliased to each other at location loc in context $\mathrm{Con}_{2}^{\prime}$. Similarly, we may show that if pointers $p^{\prime}$ and $q^{\prime}$ are aliased in $\mathrm{Con}_{2}^{\prime}$, then they are also aliased in $\mathrm{Con}_{1}^{\prime}$. Thus the set of aliases of each pointer in $C l(R)$ are the same in $\operatorname{Con}_{1}^{\prime}$ and $\operatorname{Con}_{2}^{\prime}$.

Since the set $R$ contains all the lock pointers in the given program, we have that the (must-)aliases of all lock pointers are the same in the two contexts $\operatorname{Con}_{1}^{\prime}$ and $\operatorname{Con}_{2}^{\prime}$. Also, by our hypothesis, the must-locksets are the same at locations $l_{\text {call }}$ and $l_{\text {call }}$ in contexts $\mathrm{Con}_{1}$ and $\mathrm{Con}_{2}$, respectively. Furthermore in extending the contexts $\operatorname{con}_{1}$ and $\operatorname{con}_{2}$ to form $C o n_{1}^{\prime}$ and $C o n_{2}^{\prime}$, respectively, the same locks will be acquired and released during the calls $f c_{1}$ and $f c_{2}$. Thus by combining the above facts, we have that the mustlocksets at each location loc of $h$ will be the same in both the contexts $\mathrm{Con}_{1}^{\prime}$ and $\mathrm{Con}_{2}^{\prime}$.

QED.

Let $P$ be the Steensgaard closed set $C l(S)$, where $S$ is the set of lock, thread and function pointers. The above result implies that if during the construction of the CCFG a function call fcall is encountered again in a context con, then it suffices to explore $f$ call only if either (i) the set of aliases for some pointer $p \in P$, or (ii) the lockset, is different from the instances of fcall encountered before.

Our procedure is a worklist-based algorithm shown as Algorithm 1. Starting from the entry location of the given concurrent program, we build a graph over tuples of the form (con, func, loc, $\mathcal{D P}$ ), where loc is the current program location of function func in context con. Here con is defined by means of a call string, i.e., a sequence of function calls leading to func. The alias sets and locksets are tracked via the dataflow tuple $\mathcal{D} \mathcal{P}=(\mathcal{A}, L)$, where (i) the aliasing relation $\mathcal{A} \subseteq P \times 2^{P}$ assign to each pointer $p \in P$ the set $\mathcal{A}(p)$ of aliases of $p$ at location loc of function func in context con, and (ii) $L$ is the must-lockset at location loc of function func in context con.

To start with the alias set for each pointer $p$ is set to $\{p\}$ and the lockset is set to the empty set. In each iteration, we delete a tuple tup $=($ con, func, loc, $\mathcal{D} \mathcal{F})$ from worklist $W$, where $\mathcal{D} \mathcal{F}=(\mathcal{A}, L)$. If loc has been visited before with the lockset $L$ and aliasing relation $\mathcal{A}$ then, by Thm. 1, exploring the successors of loc in con with the dataflow tuple $\mathcal{D} \mathcal{F}$ cannot lead to the discovery of a new shared variable access with a different lockset. Thus tup needs to be processed only if $l o c$ hasn't been visited before with the current set of dataflow facts (step 5 of Alg. 1).

In processing tup we consider three cases. If $l o c$ is the location of a function call fcall to function $g$, say, then in computing the successor of tup, we simply update the current location, function and context while leaving the dataflow facts unchanged (steps 7 9). If, on the other hand, the statement at $l o c$ modifies a pointer in $P$, the aliasing relation needs to be re-computed (steps 11-13). In order to avoid re-computing the aliasing relation from scratch every time we visit $l o c$ we leverage the use of summarization (see below). Similarly, if $l o c$ is the site of a locking/unlocking statement, then the must-lockset $L$ needs to be updated (steps 14-16).

Termination. In Alg. 1, each function is explored the number of times its entry location is visited with a different dataflow tuple $\mathcal{D} \mathcal{F}=(\mathcal{A}, L)$. In the worst case, the number of possible values of $\mathcal{A}$ are $|P| 2^{|P|}$, where $|P|$ is the cardinality of the Steensgaardclosed set $P$ that is input to Alg. 1. This is because from the fact that $P$ is Steensgaard-closed it follows that all aliases of a pointer $p \in P$ also belong to $P$. Thus there are at most $2^{|P|}$ different alias sets possible for $p$. Also, the number of possible locksets is at most $2^{|\mathcal{L}|}$, where $\mathcal{L}$ is the set of locks in the given program. If $|F|$ is the total number of functions, then the size of resulting CCFG is $O\left(|F||P| 2^{|P|} 2^{|\mathcal{L}|}\right)$. In practice, however, the actual locksets as well as possible aliases of thread, function and lock pointers at a given location are few in number.

A Note on Summarization. In step 12 of Alg. 1, we are required to compute the aliases of all pointers in $P$ at location loc in context con. The number of times we need to carry out this computation equals the number of different contexts in which loc is visited. In order to avoid computing the aliases from scratch each time we visit $l o c$, we makes use of summarization as formulated in [11]. Here the summary of a function $f$ tracks the start and end points of complete update sequences between pairs of locations of $f$ of the form $(m, n)$, where (i) $m$ is either the entry location of $f$ or the location of a function call in $f$, (ii) $n$ is either the exit location of $f$ or the location of a function call in $f$, and (iii) there exists a path in the (local) flow graph of $f$ from $m$ to $n$ that does not involve an intermediate function call location. When building the summaries for $f$, we do not propagate update sequences to or from functions called in $f$ because in the presence of function pointers we cannot always resolve the functions being called. Then given a context con, we compose the local update sequences along all function calls in con to compute the entire update sequences starting at the entry location of the given program to loc along con. This gives us the required aliases. It is worth noting that using bootstrapping we need to build summaries only for pointers in $C l(S)$, where $S$ is the set of lock, function and thread pointers. Since these pointers are few in number and are updated in a small number of functions, most of the function summaries will in fact be empty. Thus bootstrapping further enhances the effectiveness of summarization in computing FSCS pointer aliases efficiently.

Implicit contexts. Although Alg. 1 enumerates contexts explicitly for the sake of exposition, a practical implementation may not do so for efficiency reasons. In practice, we will number the contexts and all the data flow facts including pointer aliases and locksets will be indexed by the context number they correspond to, at each relevant program location. A context manager can be used to provide the main context-related operations, i.e., (i) context numbering, (ii) mapping contexts to data flow facts, (iii) checking equivalence of contexts, and (iv) adding new contexts for a function.

Preservation of Data Races. If the input $P$ to Alg. 1 is $C l(S)$, where $S$ is the set of lock, function and thread pointers, then we need to show that the reduced set of contexts that are generated by 


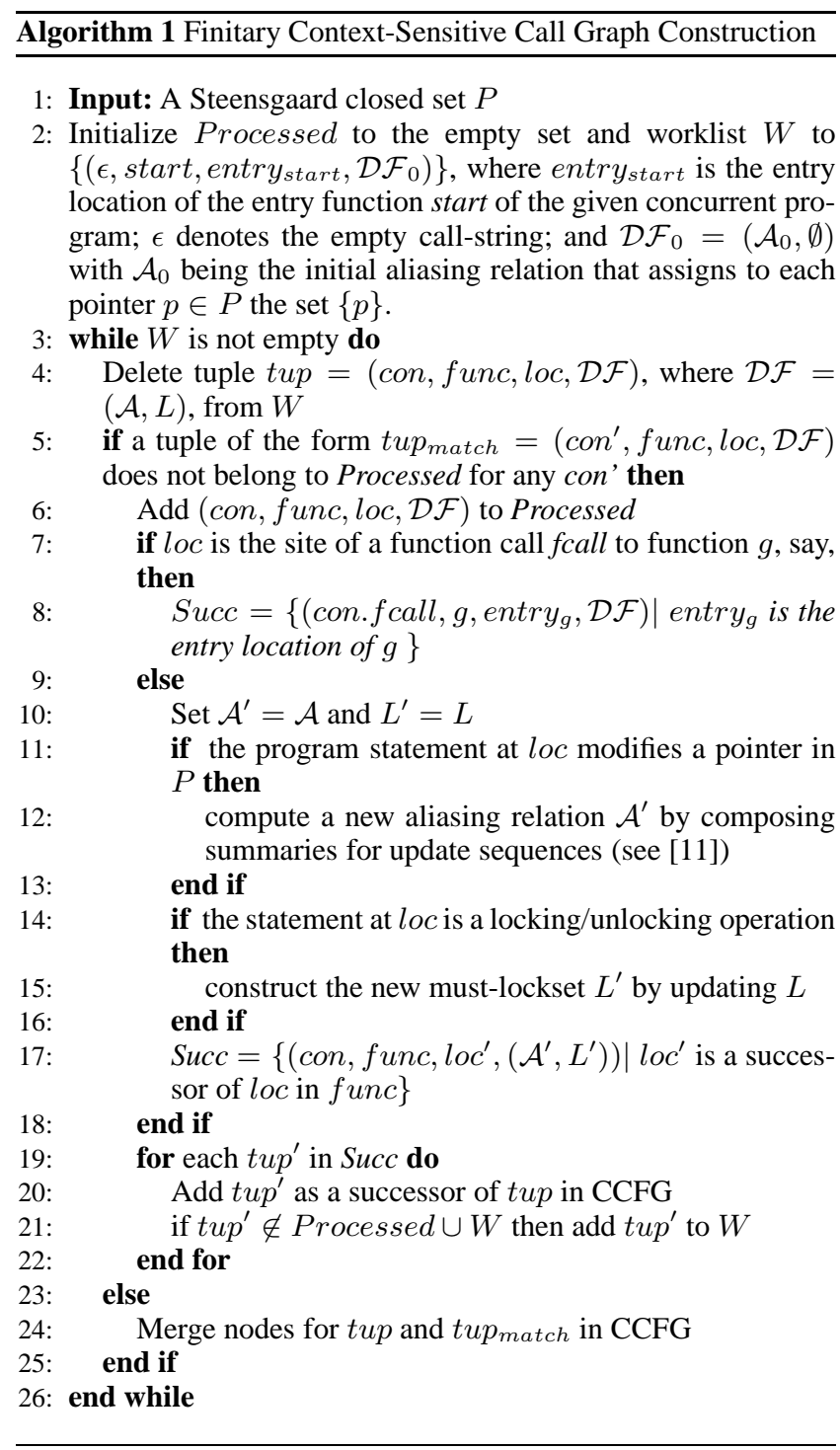

Alg. 1 does not cause us to miss any data race. Towards that end we show the following (see [26] for the proof).

Theorem 2 (Soundness). Let L be the must-lockset at location loc in a valid context con of the given program. Then there exists a valid context con' in the CCFG constructed via Alg. 1 such that the must-lockset at loc in con' is $L$.

Proof. Let $L$ be the must-lockset at location loc along a path $x$ of the given program in context con. We show that there exists a path $y$ in the CCFG leading to loc along which the must-lockset at $l o c$ is $L$. The proof is by induction on the length of $x$.

For the base case where the length of $x$ is one, $x$ is comprised only of the initial state of the given concurrent program and so the result holds trivially. For the induction step, suppose that the result holds for all paths of length less than or equal to $k$ and let $x=x_{0} x_{1} \ldots x_{k}$ be a path of length $k+1$. Let $l o c^{\prime}$ be the program location and $L^{\prime}$ the must-lockset at $x_{k-1}$ along $x$. Then by the induction hypothesis, there exists a path $y^{\prime}=y_{0} \ldots y_{l}$ of the CCFG leading to $l o c^{\prime}$ such that the must-lockset at $l o c^{\prime}$ along $y^{\prime}$ is $L^{\prime}$ and the aliases of all pointers in the Steensgaard closed set $P$ (input to
Alg. 1) at $l o c^{\prime}$ are the same along $x^{\prime}=x_{0} \ldots x_{k-1}$ and $y^{\prime}$. We now consider two cases.

First, we assume that $l o c$ ' is not a function call site, i.e., a location where a function is called. In this case we show that $y=y^{\prime} . x_{k}$ is the desired path. We consider two sub-cases. First, assume that $l o c$ is not a locking/unlocking site. Then the mustlocksets at $l o c$ ' and $l o c$ along $x$ are the same, i.e., $L^{\prime}$. Moreover, the must-lockset at $x_{k}$ along $y$ is the same as the must-lockset at $y_{l}$ along $y^{\prime}$, which by the induction hypothesis is $L^{\prime}$. If $l o c$ is a locking/unlocking statement then since the aliases of all pointers in $P$ (including the lock pointers) are the same at loc' along both $x^{\prime}$ and $y^{\prime}$ we see that the lock that is released or acquired at loc along $x$ is the same as the one that is released or acquired at loc along $y$. Thus in both cases the must-lockset at loc is the same along both $x$ and $y$.

Next, we consider the aliases of pointers in $P$. If the statement at loc does not modify any pointer in $P$, then since $P$ is a Steensgaard closed set, the set of aliases of each pointer in $P$ is the same at $l o c$ and $l o c^{\prime}$. If, on the other hand, the statement at $l o c$ ' modifies the value of some pointer in $P$, then we need to show that the set of aliases of each pointer in $P$ are the same at loc along both $x$ and $y$. Towards that end, let $p$ and $q$ be aliased to each other at $l o c$ along $x$. By the aliasing theorem (pg. 4), there exist maximal update sequences $\operatorname{seq}_{p}: l_{i_{1}}: p_{1}=a_{0}, l_{i_{2}}: p_{2}=a_{1}, \ldots, l_{i_{k}}: p_{k}=a_{k-1}$ and $\operatorname{seq}_{q}: l_{j_{1}}: q_{1}=b_{0}, l_{j_{2}}: q_{2}=b_{1}, \ldots, l_{j_{m}}: q_{m}=b_{m-1}$ starting from the entry location of the given program and leading to loc along $x$ such that $a_{0}=b_{0}$ and $p_{k}$ and $q_{m}$ are semantically equal to $p$ and $q$ at locations $l_{i_{k}}$ and $l_{j_{m}}$, respectively. We first consider the case where none of the locations $l_{i_{k}}$ and $l_{j_{m}}$ is loc. In that case $s e q_{p}$ and $s e q_{q}$ are also maximal update sequences starting from the entry location of the given program and leading to loc' along $x$. Thus $p$ and $q$ are aliased at $l o c$ ' along $x$ and hence, by the induction hypothesis, at loc' along $y$. Since the statement at loc cannot extend the update sequences that cause $p$ and $q$ to be aliased at $l o c$ ' along $y^{\prime}, p$ and $q$ are also aliased at $l o c$ along $y$. Now consider the case where at least one of $l_{i_{k}}$ or $l_{j_{m}}$ is $l o c$. Note that both $l_{i_{k}}$ and $l_{j_{m}}$ cannot be $l o c$ as only one variable can be modified at a given location. Assume for definiteness that $l_{i_{k}}=l o c$. Then consider the maximally complete update sub-sequence $s e q_{p}^{\prime}: l_{i_{1}}: p_{1}=a_{0}$, $l_{i_{2}}: p_{2}=a_{1}, \ldots, l_{i_{k-1}}: p_{k-1}=a_{k-2}$ of $s e q_{p}$. Since both $l_{i_{k-1}}$ and $l_{j_{m}}$ occur before loc along $x$ we see that $p_{k-1}$ and $q$ are aliased at loc' along $x$. Then by the induction hypothesis they are aliased at loc' along $y$. Applying the aliasing result again, we have that there exist complete maximal update sequences $\operatorname{seq}_{p}^{\prime}: l_{i_{1}^{\prime}}: r_{1}=c_{0}$, $l_{i_{2}^{\prime}}: r_{2}=c_{1}, \ldots, l_{i_{u}^{\prime}}: r_{u}=c_{u-1}$ and $\operatorname{seq}_{q}^{\prime}: l_{j_{1}^{\prime}}: s_{1}=d_{0}$, $l_{j_{2}^{\prime}}^{2}: s_{2}=d_{1}, \ldots, l_{j_{v}^{\prime}}: s_{v}=d_{v-1}$ starting from the entry location of the given program and leading to loc' along $y^{\prime}$ such that $r_{u}$ and $s_{v}$ are semantically equal to $p_{k-1}$ and $q_{m}$ (which is semantically equivalent to $q$ ) at $l_{i_{u}^{\prime}}$ and $l_{j_{v}^{\prime}}$, respectively. Then the sequence $\operatorname{seq}_{p}: r_{1}=c_{0}, l_{i_{2}^{\prime}}: r_{2}=c_{1}, \ldots, l_{i_{u}^{\prime}}: r_{u}=c_{u-1}, l_{i_{k}}: p_{k}=a_{k-1}$ is a complete update sequence from the entry location of the given program to loc along $y$. Also, $\mathrm{seq}_{q}$ is a complete update sequence from the entry location of the given program to loc along $y$. Since $p_{k}$ and $q_{m}$ are semantically equal to $p$ and $q$, respectively, we have that $p$ and $q$ are aliased at loc' along $y$.

Finally, we consider the case when $l o c$ ' is a function call site. By the induction hypothesis, the path $y^{\prime}$ occurs in the CCFG. If the path $y^{\prime} . x_{k}$ also occurs in the CCFG then we are done. Else, the only reason why $y^{\prime} \cdot x_{k}$ will not be explored is if there exists an alternative path $z$ in CCFG leading to $x_{k}$ such that the must-lockset at $x_{k}$ along $z$ is $L$ and the aliases of all pointers in $P$ are the same at loc. In that case $z$ is the desired path. This completes the induction step and proves the desired result.

QED. 


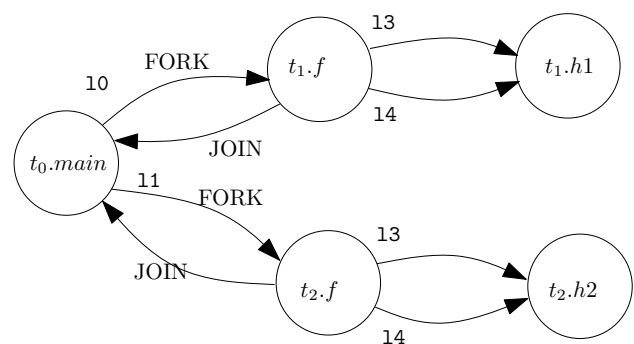

Figure 4. Concurrent call graph for the example in Fig. 1

Since, by Theorem 2, Alg. 1 preserves all possible locksets at locations where shared variables are accessed, it follows immediately that we will not miss any data race.

Example. The concurrent call graph for the example program in Figure 1 is shown in Figure 4. Note that each node in labeled by both the thread identifier and the function name. Multiple calls to other functions from a single function is labeled by the corresponding calling locations in order to distinguish the contexts. The fork and join edges are also explicitly marked.

\section{Data Race Analysis}

As mentioned in Section 5, the key steps in static data race detection are (i) computing the set of shared variables, (ii) determining the set of must-locksets at each shared variable access in a contextsensitive manner, and (iii) pruning spurious data race warnings by taking into account locksets and program causality constraints. In this section, we will discuss steps (i) and (ii) in some detail and then present a pruning strategy that relies on a may-happen-in-parallel (MHP) analysis.

Shared Variable Detection. In real-life concurrent C programs shared variables are typically accessed via pointers. The set of shared variables consists of all variables that are either global variables of threads, aliases of global variables or escaped variables. Note that local pointer variables may also alias global variables and result in a data race violation. However, if we label all local variables aliasing global variables as shared, we will end up with a large number of spurious warnings. Therefore, we are only interested in the set of local pointers that are used to actually update the values of global variables. Towards that end, using the CCFG computed in the previous section, we use a data flow analysis on pointer variables [13] to compute the set of shared variable accesses. The analysis essentially propagates the assignments in complete update sequences from variables $p$ to $q$ (where $p$ is global). If the sequence is followed a modification of some scalar variable via $q$, then $q$ is marked as shared.

Initial Data-race warnings. After we have computed the set of shared variables, we can conservatively enumerate data race warnings by considering all pairs of accesses to the same shared variable in two different threads, where at least one of the accesses is a write operation. For industrial size programs, this conservative enumeration may lead to thousands of warnings, most of which are bogus. We now describe a staged MHP analysis, which prunes away the spurious warnings by first taking into account lock acquisition patterns and then using thread ordering constraints imposed by fork and join operations in the CCFG.

\subsection{MHP analysis}

Lockset analysis. Computing context-sensitive locksets is trivial after computing the context-sensitive CCFG for the program (cf. Algorithm 1): the locksets can be extracted from the data flow facts $\mathcal{D F}$ computed at the shared variable access locations. By com- bining lockset computation with the call graph computation, we avoid a separate lockset computation phase, required in previous approaches [13]. Then two accesses in different thread may happen in parallel only if the locksets held at their respective locations are disjoint.

Thread order analysis. The order of program statements in the CCFG prohibits some concurrent accesses. For example, consider a concurrent program comprised of two threads: $t_{0}$ and $t_{1}$, where $t_{0}$ creates $t_{1}$ and later waits for $t_{1}$ to join. An access to a shared variable $x$ in thread $t_{1}$ cannot happen concurrently with an access to $x$ in $t_{0}$ if the access in $t_{0}$ follows the join instruction corresponding to $t_{1}$. The thread order analysis is designed to reveal the causalities (of above form) in a program that arise due to fork/join constraints as well as (sequential) control flow in threads.

Fork-join model. We start by observing that fork and join operations enforce the following causality constraints:

1. All statements in the parent thread between matching fork and join points may happen in parallel with all statements in the child thread (and all threads forked thereof)

2. No other statements can happen in parallel.

The thread order analysis exploits the above causality constraints in the fork-join model to compute an MHP set.

Thread Pool model. Recall that in the thread pool model, tasks are dispatched for execution to previously created threads. These calls implicitly correspond to a fork operation. However, these fork operations do not have corresponding join operations. In order to conservatively estimate the effect of these calls, we introduce matching join operations at the exit locations of the start function of the given program.

Furthermore, in contrast to the fork-join model, the thread pool model (by virtue of the work queue) allows multiple function tasks to be executed on the same thread. Since only one task can be executing on a thread at any given time, we add the constraint that no two functions (and any pair of locations in those functions) which always execute on the same thread may happen in parallel. Note that the information about the set of functions that always execute on the same thread is readily available from the CCFG.

Given a thread $t$, let children $(t)$ denote the set of threads created by $t$. For each thread $t$, we denote the first and last program locations in $t$ by $t$.start and $t$.end respectively. In general, t.start and $t$.end are not unique since multiple fork calls can be dispatched to the same thread in the thread pool model. However, given a fork call instruction to thread $t$, t.start and $t$.end are uniquely defined. For program locations $l$ and $m$, we write $l \rightarrow_{f j} m$ to mean that there exists a path in the CCFG of the given program from $l$ to $m$. Similarly, $l \rightarrow m$ means that there exists a path in the CCFG from $l$ to $m$ along which there exists no fork or join statements.

Algorithm 2 shows the details of the analysis. Intuitively, Loc parent consists of all the locations in the parent thread that are forward reachable from the fork call and backward reachable from the join instruction without any intermediate fork or join edges. The set $L o c_{\text {child }}$ consists of all locations in the child thread as well as threads created thereof. The analysis produces the set MHPlocs of location pairs that may happen in parallel.

Based on the thread order analysis, we can now prune the warnings by removing any warning wherein the program location pairs that do not occur in the MHPlocs set.

Example. Recall the example program in Figure 1. As mentioned earlier, the program has two potential data race conditions in the function $f$ on the shared variable pointed to by the argument $z$. More precisely, the races involve location pairs ( $t 1 . f . l 2, t 2 . f . l 2)$ and ( $t 1 . f . l 3, t 2 . f . l 3)$ since function $f$ is executed by both threads $t 1$ and $t 2$. However, if we perform thread order analysis on the context-sensitive CCFG obtained from the program, we find that 


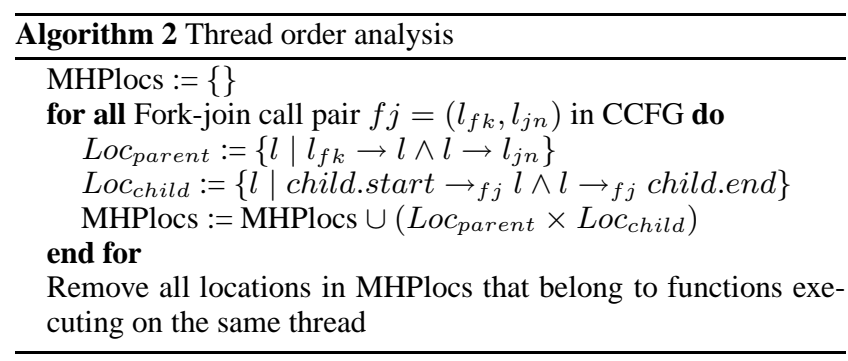

the above location pairs are not in the set MHPlocs, since the thread $t 2$ can only execute after the thread $t 1$ finishes executing.

\section{Experiments}

The proposed data race detection technique has been implemented in the CoBE framework [12] for analysis of concurrent $C$ programs being developed at NEC. All the experiments were performed on an Intel Core Duo $1.86 \mathrm{Ghz}$ machine with $1 \mathrm{~GB}$ memory, running Linux. In order to evaluate the usefulness of the proposed approach, we applied it to a wide variety of software: open source benchmarks including Linux drivers, bzip2smp software, and in-house products including a parallel MPEG decoder $\mathbf{D}$ and a commercial software system, $\mathbf{S}$. Table 1 shows the experimental results. The system $\mathbf{S}$ was the prime inspiration behind this work since it is based on the thread pool model and employs AFCs as well as locks for synchronization, and also contains recursive functions. The other three benchmarks are chosen to show the effectiveness of the proposed CCFG construction method over the previous method [13], even on programs without function pointers or recursion.

Linux Drivers: In previous work [13], we reported data race detection statistics on a suite of Linux drivers with known data races downloaded from kernel.org. The goal of this exercise was to test whether CoBE could detect these known data races. We showed that CoBE could in fact discover all the known data races efficiently while keeping the bogus warnings rate low. The procedure formulated in [13] carried out data race detection by enumerating all contexts in the given program and computing locksets individually for each context, without checking for context equivalence with respect to locksets. The time taken for static warning generation via exhaustive context enumeration is given in col. Ex-Time of Table 1. Using our new CCFG construction that exploits the fix-point criterion to drastically cut down on the contexts that need be explored, we see that the time taken (shown in LFC-Time col.) for data race detection is reduced many-fold. Thus, although the fix-point criterion was proposed for finitizing the CCFG in the presence of function pointers and recursion, it also enhances the scalability of existing approaches by considering only a representative set of contexts necessary for data-race detection. Cols. \#War and \#Aft. Red. refer, respectively, to the initial number of warnings generated via the lockset method and the number of warnings left after applying warning reduction which combines thread order analysis (Sec. 7.1) with existing reduction schemes [13].

bzip2smp: The bzip2smp program parallelizes the bzip compression algorithm to work on SMP machines. It uses the pthread library as opposed to AFCs. Thus our new framework can handle both the fork/join and the thread pool models in a unified manner.

Parallel Decoder D: D is an in-house parallel implementation of an MPEG-4 decoder. Notice the stark difference between the time taken in exploring all the contexts exhaustively as in [13] and in exploring the contexts enumerated via our new CCFG construction. The dramatic difference is because for efficiency reasons the implementation does not use any locks; all synchronization is via the use of barriers. Thus each function $f$ is explored only once during our CCFG construction (corresponding to a single lockset data fact, see Algorithm 1) as opposed to [13] where it is explored for each different context in which $f$ appears.

NEC Product S: a large in-house concurrent software system, denoted by $\mathbf{S}$. The $\mathbf{S}$ system consists of about $400 \mathrm{~K}$ lines of $\mathrm{C}++$ code using Boost libraries [23] and is based on a thread pool model. Much of the code deals with asynchronous stream processing, which makes execution flow non-evident. Callback objects are passed as first-class data items. The final execution context for any particular callback object is not evident and often is the source of subtle errors. Changes made in one small routine have often led to bugs occurring much later in code, at a location seemingly unrelated to the original bug location. These bugs are mostly irreproducible due to the complex nature of asynchronous calls and remain unexpressed over long periods of time.

We evaluated our approach by checking for data races in a central module $M$ of $\mathbf{S}$ (about $10 \mathrm{~K}$ of $\mathbf{C}++$ code). To this goal, we first built a faithful $\mathrm{C}$ model of $M$. This primarily involved recreating the thread pool model based on the pthread library and building the infrastructure for programming asynchronous function calls (including modeling the bind functionality of the Boost library). Based on this infrastructure, we were able to obtain a $\mathrm{C}$ model of the module $M$ that closely resembled the actual $\mathrm{C}++$ implementation. We performed data-race detection on this model: we observed that the initial number of data race warnings with the context-sensitive CCFG construction and the lockset based reduction were 524. Exploiting the thread order analysis drastically reduced the number of warnings to 161 . Note due to bootstrapping the time taken to analyze this code was only $29 \mathrm{sec}$. This would not have been possible if we had carried out a whole program FSCS points-to analysis. Thus bootstrapping was key in making our analysis scalable.

\section{Related Work and Conclusions}

Data race detection being a problem of fundamental interest has been the subject of extensive research. Many techniques have been proposed in order to attack the problem including dynamic run-time detection, static analysis and model checking.

Early work on dynamic data race detection includes the Eraser data race detector [22] which is based on computing locksets. There has been much work that improves upon the basic Eraser methodology. One such approach is [9] which leverages the use of static analysis to reduce the number of data race warnings that need to be validated via a run-time analysis. Other run-time detection tools based on Lamport's happened before model restrict the number of interleavings that need be explored $[5,16]$. The advantage of runtime techniques is the absence of false warnings. On the other hand, the disadvantages are the extra cost incurred in instrumenting the code and poor coverage both of which become worse as the size of code increases especially in the context of concurrent programs. Additionally, run time detection techniques presume that the give code can be executed which may not be an option for applications like device drivers.

Recently, there has been a spurt of activity in applying static analysis techniques for data race detection $[4,14,25,2,18,17,7$, $20,9]$. An advantage of such techniques is that they can be made to scale to large programs. The key disadvantage is that since static analysis works on heavily abstracted versions of the original program, they are not refined enough and can produce a large number of false warnings. Concurrency or MHP analysis has received wide attention [21] for object-oriented programs (see, e.g., [27, 1]). These analyses rely on the fact that at thread creation/join locations, the target thread object/code can be syntactically inferred. In 


\begin{tabular}{|l||c|c|c|c|c|c|}
\hline \hline Driver & KLOC & \# ShVars & \#War & \#Aft.Red. & Ex-Time (secs) & LFC-Time (secs) \\
\hline \hline hugetlb & 1.2 & 5 & 4 & 1 & 3.2 & 1 \\
\hline ipoib_multicast & 26.1 & 10 & 33228 & 6 & 7 & 1.4 \\
\hline plip & 13.7 & 17 & 94 & 51 & 5 & 3 \\
\hline sock & 0.9 & 6 & 32 & 13 & 2 & 1.2 \\
\hline ctrace_comb & 1.4 & 19 & 985 & 58 & 6.7 & 1.1 \\
\hline autofs_expire & 8.3 & 7 & 20 & 3 & 6 & 1.9 \\
\hline ptrace & 15.4 & 3 & 9 & 1 & 15 & 2.4 \\
\hline tty_io & 17.8 & 1 & 6 & 3 & 4 & 0.9 \\
\hline raid & 17.2 & 6 & 23 & 13 & 1.5 & 1.1 \\
\hline pci_gart & 0.6 & 1 & 3 & 1 & 1 & 0.8 \\
\hline \hline bzip2smp & 6.4 & 25 & 15 & 12 & - & 23 \\
\hline \hline D (NEC) & 2.9 & 4 & 256 & 25 & $22 \mathrm{~min}$ & $8 \mathrm{sec}$ \\
\hline \hline S (NEC) & 1.3 & 12 & 524 & 161 & - & $29 \mathrm{sec}$ \\
\hline
\end{tabular}

Table 1. Experimental comparison of data-race detection on benchmarks. The column Ex.Time reports the time taken (in seconds) via the exhaustive context enumeration algorithm of [13] whereas the LFC-Time column denotes the time taken for exploring the reduced set of contexts enumerated by our new CCFG construction. The '-' entries in the table indicate that the previous technique [13] could not handle named forks as a result of which experiments could not be carried out on these examples.

contrast, this paper analyzes programs where thread dispatch takes place via indirect calls through function pointers. Moreover, since the exact target depends on the particular calling context, performing race detection on a context-sensitive control flow graph reduces the number of spurious data races significantly.

Verification of particular forms of asynchronous programs has received some attention recently $[10,8]$. In both cases, however, asynchronous tasks are executed sequentially and there is no concurrent interaction between the tasks. To the best of our knowledge, no previous method has investigated data race detection for general asynchronous multi-threaded programs with indirect calls.

Emami et al. [6] present an algorithm for context-sensitive interprocedural points-to analysis for recursive sequential programs with calls made via function pointers. However, handling recursion via the introduction of back-edges results in an over-approximation of the set of behaviors of the given program. As a result, the pointsto analysis no longer remains fully context-sensitive - a key requirement for static data race detection - leading to bogus warnings. Our key insight, on the other hand, is that even in the presence of recursion and concurrency, it is possible to perform a precise and efficient data race detection by considering a finite representative subset of contexts (instead of over-approximating the set of contexts) for each function to precisely identify the shared variables as well as compute context-sensitive locksets and points-to sets of function/thread pointers for CFG construction.

In this paper, we have presented a method to perform static data race detection for concurrent $\mathrm{C}$ programs that use asynchronous indirect function calls for communication - a problem of great practical importance but which has received little attention in the literature. One of our key contributions has been a new technique for context-sensitive CFG construction that guarantees termination even in the presence of recursion and without losing precision of the analysis at hand. This enables us to build a framework for fast and accurate data race detection that can handle concurrent programs with complex programming constructs thereby making such an analysis practical for a larger class of realistic programs. Experiments on real-life open source benchmarks have demonstrated the efficacy of our new technique.

\section{References}

[1] R. Barik. Efficient computation of may-happen-in-parallel information for concurrent java programs. In $L C P C$, pages 152-169, 2005.
[2] M. Burrows and K. Leino. Finding stale-value errors in concurrent programs. In Compaq Systems Research Center SRC-TR-2002-004, 2002.

[3] P. Chandrasekaran, C. L. Conway, J. M. Joy, and S. K. Rajamani. Programming asynchronous layers with clarity. In FSE, 2007.

[4] D. Detlefs, K. Leino, G. Nelson, and J. Saxe. Extended static checking. In TR SRC-159 Compaq SRC, 1998.

[5] A. Dinning and E. Schonberg. An empirical comparision of monitoring algorithms for access anomaly detection. In PPoPP, 1990.

[6] M. Emami, R. Ghiya, and L. J. Hendren. Context-sensitive interprocedural points-to analysis in the presence of function pointers. pages 242-256, 1994.

[7] D. Engler and K. Ashcraft. RacerX: Effective, Static Detection of Race Conditions and Deadlocks. In SOSP, 2003.

[8] P. Ganty, R. Majumdar, and A. Rybalchenko. Verifying liveness for asynchronous programs. In POPL, pages 102-113, 2009.

[9] C. J, K. Lee, A. Loginov, R.O'Callahan, V. Sarkar, and M. Sridharan. Efficient and precise datarace detection for multithreaded objectoriented programs. In PLDI, 2002.

[10] R. Jhala and R. Majumdar. Interprocedural analysis of asynchronous programs. In POPL, 2007.

[11] V. Kahlon. Bootstrapping: A technique for scalable flow and contextsensitive pointer alias analysis. In PLDI, 2008.

[12] V. Kahlon, S. Sankaranarayanan, and A. Gupta. Semantic reduction of thread interleavings for concurrent programs. In TACAS, 2009.

[13] V. Kahlon, Y. Yang, S. Sankaranarayanan, and A. Gupta. Fast and accurate static data-race detection for concurrent programs. In $C A V$, 2007.

[14] R. Leino, G. Neslon, and J. Saxe. Esc/java users' manual. In Technical Note 2000-002, Compaq Systems Research Center, 2001.

[15] P. Li and S. Zdancewic. Combining events and threads for scalable network services implementation and evaluation of monadic, application-level concurrency primitives. In PLDI, 2007.

[16] J. Mellor-Crummey. One-the-fly detection of data races for programs with nested fork-join parallelism. In Proceedings of the 1991 Supercomputer Debugging Workshop, 1991.

[17] M. Naik and A. Aiken. Conditional must not aliasing for static race detection. In POPL, 2007.

[18] M. Naik, A. Aiken, and J. Whaley. Effective static race detection for java. In PLDI, 2006.

[19] C. Pheatt. Intel $®$ threading building blocks. J. Comput. Small Coll., 23(4):298-298, 2008.

[20] P. Pratikakis, J. S. Foster, and M. Hicks. LOCKSMITH: Context- 
Sensitive Correlation Analysis for Race Detection. In PLDI, 2006.

[21] M. C. Rinard. Analysis of multithreaded programs. In SAS, 2001.

[22] S. Savage, M. Burrows, G. Nelson, P. Sobalvarro, and T. Anderson. Eraser: A dynamic data race detector for multithreaded programming. In ACM TCS, volume 15(4), 1997.

[23] J. G. Siek, L.-Q. Lee, and A. Lumsdaine. The Boost Graph Library User Guide and Reference Manual (With CD-ROM). Addison-Wesley Professional, December 2001.

[24] B. Steensgaard. Points-to analysis in almost linear time. In POPL, 1996.

[25] N. Sterling. Warlock: A static data race analysis tool. In USENIX Winter Technical Conference, 1993.

[26] F. Version. www.cs .utexas .edu/users/kahlon/async.

[27] C. von Praun and T. R. Gross. Static conflict analysis for multithreaded object-oriented programs. SIGPLAN Not., 38(5):115-128, 2003.

[28] M. Welsh, D. Culler, and E. Brewer. Seda: an architecture for wellconditioned, scalable internet services. SIGOPS Oper. Syst. Rev, 35(5):230-243, 2001. 